\title{
The Potential Cost Effectiveness of Different Dengue Vaccination Programmes in Malaysia: A Value-Based Pricing Assessment Using Dynamic Transmission Mathematical Modelling
}

\author{
Asrul Akmal Shafie ${ }^{1} \cdot$ Hui Yee Yeo ${ }^{1} \cdot$ Laurent Coudeville $^{2} \cdot$ Lucas Steinberg $^{3}$ • \\ Balvinder Singh Gill ${ }^{4} \cdot$ Rohani Jahis ${ }^{4} \cdot$ Amar-Singh HSS $^{5}$
}

Published online: 15 February 2017

(C) Springer International Publishing Switzerland 2017

\begin{abstract}
Background Dengue disease poses a great economic burden in Malaysia.

Methods This study evaluated the cost effectiveness and impact of dengue vaccination in Malaysia from both provider and societal perspectives using a dynamic transmission mathematical model. The model incorporated sensitivity analyses, Malaysia-specific data, evidence from recent phase III studies and pooled efficacy and long-term safety data to refine the estimates from previous published studies. Unit costs were valued in \$US, year 2013 values.
\end{abstract}

Electronic supplementary material The online version of this article (doi:10.1007/s40273-017-0487-3) contains supplementary material, which is available to authorized users.

\section{Asrul Akmal Shafie}

aakmal@usm.my

1 Discipline of Social and Administrative Pharmacy, School of Pharmaceutical Sciences, Universiti Sains Malaysia (USM), 11800 George Town, Penang, Malaysia

2 Sanofi Pasteur SA, 2 Avenue Pont Pasteur, 69367 Lyon Cedex 07, France

3 Sanofi Pasteur Malaysia, Unit TB-18-1, Level 18, Tower B, Plaza 33, No. 1 Jalan Kemajuan, Seksyen 13, 46200 Petaling Jaya, Selangor, Malaysia

4 Disease Control Division, Ministry of Health Malaysia, Block E1, E3, E6, E7 and E10, Parcel E, Federal Government Administration Centre, 62590 Putrajaya, Malaysia

5 Pediatric Department and Clinical Research Center, Hospital Raja Permaisuri Bainun Ipoh, Jalan Hospital, 30990 Ipoh, Perak, Malaysia
Results Six vaccination programmes employing a threedose schedule were identified as the most likely programmes to be implemented. In all programmes, vaccination produced positive benefits expressed as reductions in dengue cases, dengue-related deaths, life-years lost, disability-adjusted life-years and dengue treatment costs. Instead of incremental cost-effectiveness ratios (ICERs), we evaluated the cost effectiveness of the programmes by calculating the threshold prices for a highly cost-effective strategy [ICER $<1 \times$ gross domestic product (GDP) per capita] and a cost-effective strategy (ICER between 1 and $3 \times$ GDP per capita). We found that vaccination may be cost effective up to a price of \$US32.39 for programme 6 (highly cost effective up to \$US14.15) and up to a price of \$US100.59 for programme 1 (highly cost effective up to \$US47.96) from the provider perspective. The cost-effectiveness analysis is sensitive to under-reporting, vaccine protection duration and model time horizon.

Conclusion Routine vaccination for a population aged 13 years with a catch-up cohort aged 14-30 years in targeted hotspot areas appears to be the best-value strategy among those investigated. Dengue vaccination is a potentially good investment if the purchaser can negotiate a price at or below the cost-effective threshold price. 


\section{Key Points for Decision Makers}

Dengue vaccination in Malaysia reduces the denguerelated health and economic burden by $54-68 \%$ for different vaccination strategies.

In a targeted hotspot strategy that routinely vaccinates children aged 13 years with a catch-up cohort aged 14-30 years, a dengue vaccination programme would be highly cost effective from the provider perspective at a price below \$US47.96 per dose (95\% confidence interval 33.90-65.21)

Our study provides important information on the value of dengue vaccination and fair vaccine prices to aid policy makers evaluate the possibility of incorporating a dengue vaccine into the national immunization programme

\section{Introduction}

Dengue is the most common mosquito-borne disease affecting human populations. The World Health Organization (WHO) reported a 30 -fold increase in annual dengue cases in the last 50 years, with a mortality rate of $2.5 \%$ among severe dengue cases. Approximately 3.9 billion people globally are at risk, with 390 million infections occurring annually and 68\% of cases occurring in Asia [1]. Malaysia has been experiencing a surge of dengue cases in recent years, more than doubling from 43,346 cases in 2013 to 111,285 cases in 2015 [2]. In addition, studies have also shown that dengue cases in Malaysia have been underreported [3, 4].

Dengue presents an enormous economic burden to Malaysia, costing approximately 359 million Malaysian ringgits (RM) annually [3]. Currently, the only method available to control transmission is through vector control interventions as there are no specific treatments or licensed vaccines in Malaysia to protect against the disease. Furthermore, vector control has been shown to be only partially effective in reducing disease burden [5]. Six dengue vaccines are currently undergoing various stages of clinical development and are projected to be available in Malaysia by 2017-2020. In view of this rapid development, a country-specific economic evaluation of the dengue vaccine is urgently needed [6]. Information about the value of incorporating a dengue vaccination programme in current dengue control strategies are required to inform policy makers for future vaccine roll out and resource allocation.

We conducted a literature review in PubMed/MEDLINE and Web of Science databases (January 2000-September
2016) to identify and summarize the current state-of-the-art of the cost effectiveness of dengue vaccines. Five studies evaluating the cost effectiveness of dengue vaccine in the Philippines, Brazil, Thailand, Singapore and Southeast Asia were retrieved and reviewed [7-11]. All the studies found that vaccination reduced the overall dengue-related health and economic burden and was cost effective at a price between \$US0.50-270 per dose. However, despite the existing literature, the cost effectiveness of the currently available vaccine has yet to be evaluated in Malaysia. Furthermore, all the models evaluated the vaccine's cost effectiveness based on hypothetical vaccine efficacy without empirical evidence.

This study aims to evaluate the cost effectiveness and impact of dengue vaccination in Malaysia by examining different vaccination programmes using a previously published dynamic transmission mathematical model [12]. This study incorporated a series of sensitivity analyses using new clinical evidence from two recent large-scale phase III studies $[13,14]$, pooled vaccine efficacy data and long-term safety data $[15,16]$ to refine the vaccine efficacy and safety estimates from previously published studies. In addition, the Strategic Advisory Group of Experts (SAGE) on immunization from the WHO recently used the model to develop their report with recommendations for the CYDTDV vaccine, which was recently licensed in 13 countries $[17,18]$. We believe that the information provided by this paper could supplement the conclusions reported by the WHO-SAGE and benefit not only Malaysia but other countries with the same public health issue.

\section{Methods}

\subsection{Study Design and Model Description}

Our study employed a previously described dynamic transmission mathematical model [see the Electronic Supplementary Material (ESM) 1] [12] to evaluate the potential impact and cost effectiveness of dengue vaccination programmes in Malaysia compared with no vaccination. This is an age-structured, host-vector and serotypespecific deterministic compartmental model that aims to reproduce the dynamics of dengue transmission and can be used to identify the optimum vaccination age and compare the benefits of various vaccination strategies based on routine and/or mass catch-up vaccination strategies. The model also includes seasonality and accounts for the transmission dynamics of the four dengue serotypes in human and mosquito populations. The susceptible-infected-recovered (SIR) model considered ten different states for the host population. Two types of immunological interactions between serotypes can be considered with this 
model, as follows: temporary or permanent cross-protection, cross-enhancement, or their combination. In the absence of vaccination, individuals become infected after a bite from an infected mosquito. Each infection is serotype specific and provides lifelong serotype-specific immunity. The level of infection of human hosts after being bitten by a vector can vary between severe, mild and asymptomatic and depends on the viral load and intrinsic characteristics of the host. The seasonal variation in mosquito density was accounted for by varying the monthly growth rate of the vector population around an annual average.

\subsection{Model Calibration to Malaysia-Specific Epidemiological Data}

The model parameters were defined and calibrated with data specific for Malaysia (age-specific annual incidence, case fatality rate and demographic data) and empirical evidence (age-specific seroprevalence and disease impact). The calibration procedure employed two main steps and was verified using the least squares minimization method. The quality of the calibrated model was further validated by comparing the observed and simulated data and through a series of focus group discussions. The calibrated model was assessed by a dengue expert panel in Malaysia and found to be suitable to develop a satisfactory representation of the disease process. More details on validation of the model and data inputs are contained in the ESM 2.

\subsection{Vaccination Programme Evaluations and Parameter Estimations}

To increase the relevance of our studies to local policy makers, we conducted a series of focus group discussions with key stakeholders, including key Ministry of Health $(\mathrm{MOH})$ personnel to identify and prioritise the most likely dengue vaccination programmes to be implemented in Malaysia. Two focus group discussions with team members from the Disease Control Division of the $\mathrm{MOH}$ Malaysia headed by the director of the division and two face-to-face personal interviews with the head of the vaccine prevention of disease department were conducted to elicit the model inputs for the simulation of the vaccination programmes. The programme characteristics discussed were the target age group for routine and catch-up vaccination, target geographical area, vaccination coverage rate, vaccination compliance rate and possible vaccine wastage. The expert panel validated the data in a published study [19] that found the highest proportion of dengue disease cases occurred in people aged 10-29 years. Therefore, three age groups were identified as the target age groups for the programmes: $13-30,9-30$ and 9-17 years. Six vaccination programmes were identified as the most likely strategies to be implemented in Malaysia. The characteristics and parameters of these programmes are summarised in Table 1. Three programmes examined the impact of vaccination in targeted hotspots (THS) of six districts in Malaysia with the highest epidemiology burden and priority, and the other three examined the nationwide (NW) vaccination setting. As compliance, cost and coverage differed between settings, vaccination programmes were further subdivided into school-based (vaccinees aged 9-17 years) and community-based (vaccinees aged 18-30 years). Coverage and compliance for school-based programmes were estimated at 95 and 90\%, respectively, in accordance with Malaysia childhood vaccine coverage statistics [20]. However, the expert panel estimated that coverage and compliance for community-based programmes would be only $50 \%$. Vaccine wastage was estimated at $20 \%$ [21].

Vaccine characteristics and efficacy obtained from pooled data analysis [16] of the trials CYD14 and CYD15 were used to populate the model. Consequently, our evaluation considered the same tetravalent vaccine investigated in these trials. The vaccine was administered in a threedose regimen (baseline and 6 and 12 months). To remain conservative, the durations of vaccine protection were assumed to be $2.5,5$ and 10 years after vaccination completion with dose 1, 2 and 3, respectively.

We considered the costs from both the public provider and the societal perspectives. Treatment costs for a dengue case were obtained from relevant published reports and papers [3, 22, 23]. The total cost of dengue disease with the vaccination program is the sum of cost of dengue treatment and cost of vaccination. The total cost of dengue disease without the vaccination program only included dengue treatment costs. Costs estimated from the provider perspective exclude patient or family sector costs (indirect costs), and costs estimated from the societal perspective include all sector costs. The dengue treatment costs per hospitalised case were \$US672.51 and \$US933.78 and per ambulatory case were \$US326.51 and \$US456.13 from the provider and societal perspectives, respectively (Table 1) $[3,22,23]$. The indirect cost calculations included productivity losses of both the patient and relatives, and the corresponding days of school and work days based on the age distribution of cases. The indirect ambulatory and hospitalized costs of a dengue case were \$US193.28 and \$US219.79, respectively. To better represent immunization practices in Malaysia, we assumed the dengue vaccination would be fully subsidized by the government. The direct vaccination programme cost per dose from the societal perspective would therefore be the same as that from the provider perspective, i.e. \$US3.58. The indirect costs arising from vaccine administration would only include the productivity losses and the corresponding days of school 
A. A. Shafie et al

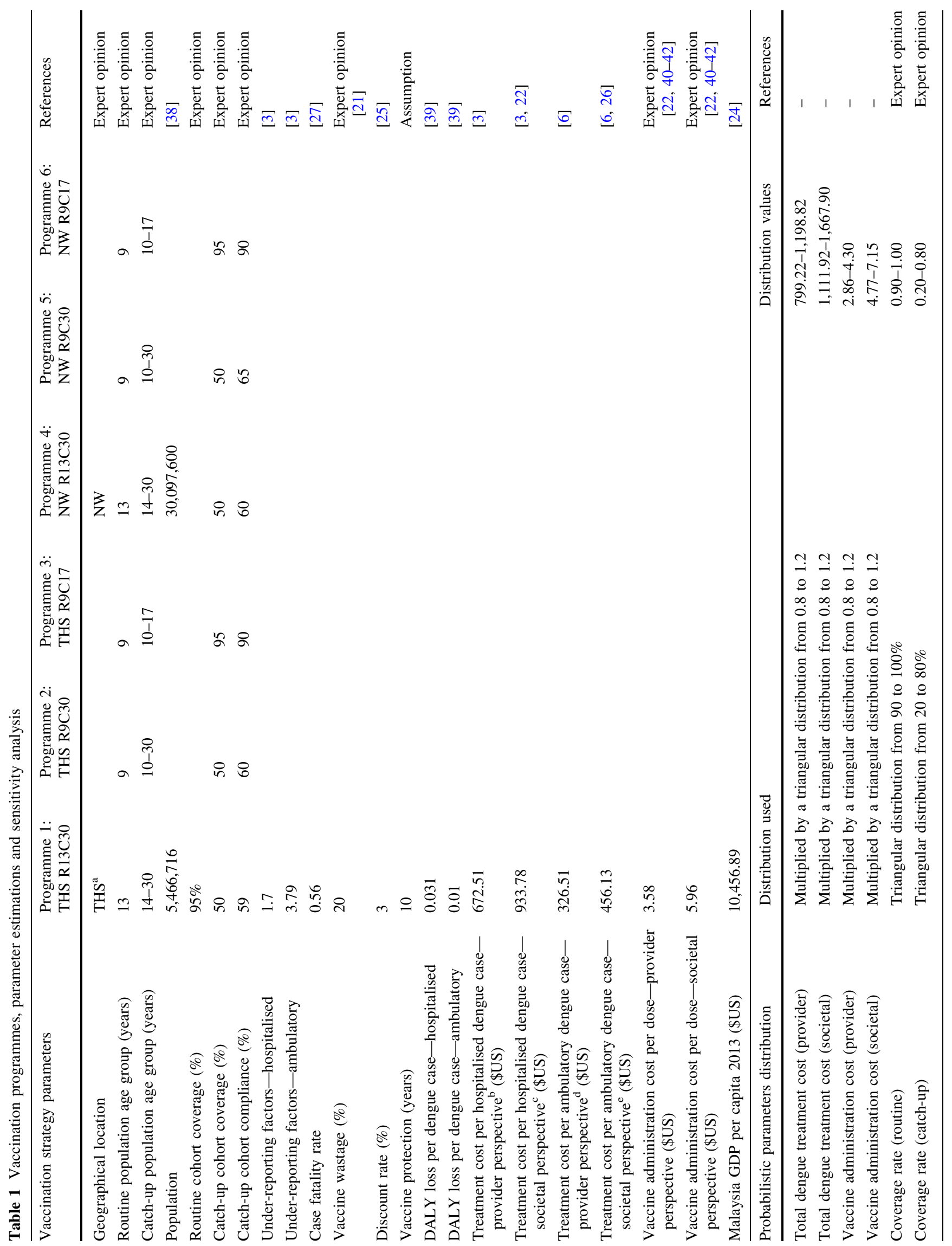




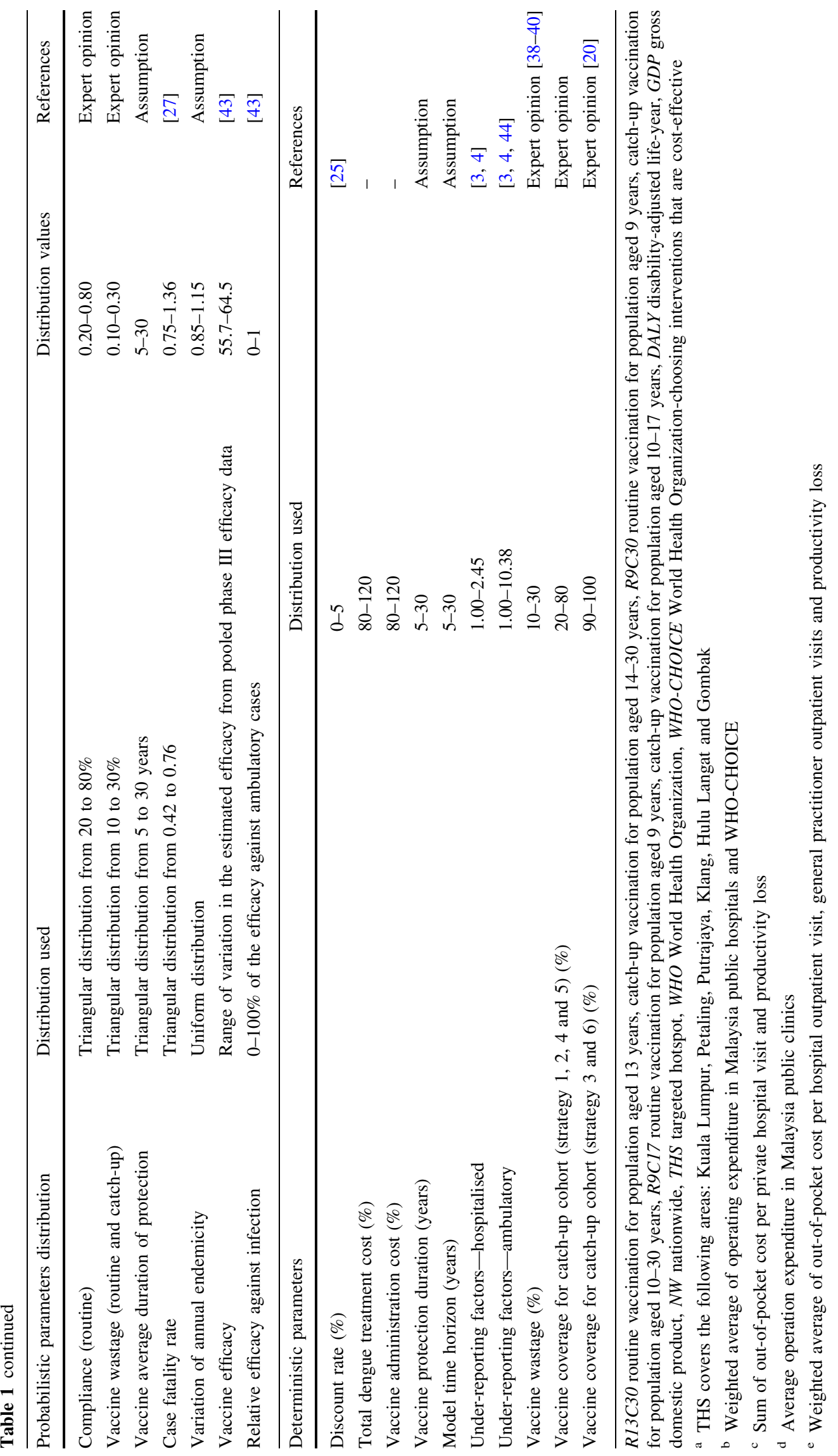


and work days based on age distribution. The weighted average indirect vaccine administration cost per dose was calculated as \$US2.38. The calculated overall vaccine administration costs per dose were therefore \$US3.58 and \$US5.96 from the provider and societal perspectives, respectively (Table 1). More details on dengue treatment and vaccine administration costs are included in the ESM 2.

All unit costs in this study were valued in \$US and inflated to 2013 values using a gross domestic product (GDP) deflator [24].

\subsection{Outcome Measures: Disease Burden, Economic Burden and Vaccine Cost Effectiveness}

Both the disease burden and the economic burden averted by vaccination were calculated by the absolute difference between vaccination and no vaccination simulations over a 10-year period (2016-2025). The outcome measures were the number of ambulatory and hospitalised dengue cases prevented, dengue-related deaths avoided, life-years lost (LYL) averted, disability-adjusted life-years (DALYs) averted, ambulatory dengue treatment costs reduced and hospitalised dengue treatment costs reduced. The economic burden was evaluated from both public provider and societal perspectives. Productivity losses were included in the calculation when examined from the societal perspective. All future costs and outcomes in this study were discounted at $3 \%$ [25].

We evaluated the cost effectiveness of the vaccination using a value-based pricing method. We presented the results as the vaccine cost-effective threshold price instead of incremental cost-effectiveness ratio (ICER). The ICER was fixed at the threshold value according to the criterion recommended by the WHO, where an intervention was classified as cost effective when the ICER was between 1 and $3 \times$ GDP per capita (\$US10,456.89-31,370.67 per DALY averted in Malaysia) and highly cost effective when the ICER was $<1 \times$ GDP per capita ( $<$ \$US10,456.89 per DALY averted in Malaysia) [24, 26]. The vaccine threshold price was solved as follows:

$P_{\text {threshold }}=\frac{\left(L_{\mathrm{R}}-L_{\mathrm{S}}\right) \times T_{\mathrm{CE}}+\left(C_{\mathrm{R}}-C_{\mathrm{S}}\right)+\left(C V_{\mathrm{R}}-C V_{\mathrm{S}}\right)}{V_{\mathrm{S}}-V_{\mathrm{R}}}$

where $C_{\mathrm{S}}$ is the discounted disease cost under the vaccination simulation over 10 years, $C_{\mathrm{R}}$ is the discounted disease cost under the no vaccination simulation over 10 years, $C V_{\mathrm{S}}$ is the discounted vaccine administration cost under the vaccination simulation over 10 years, $C V_{\mathrm{R}}$ is the discounted vaccine administration cost under the no vaccination simulation over 10 years, $V_{\mathrm{s}}$ is the discounted number of vaccinations required under the vaccination simulation over 10 years, $V_{\mathrm{R}}$ is the discounted number of vaccinations required under the no vaccination simulation over 10 years, $L_{\mathrm{S}}$ is the lost DALYs or LYLs under the vaccination simulation over 10 years, $L_{\mathrm{R}}$ is the lost DALYs or LYLs under the no vaccination simulation over 10 years and $T_{\mathrm{CE}}$ is the cost-effectiveness threshold.

\subsection{Sensitivity to Parameter Uncertainty}

In addition to assessing various vaccination programmes, we performed a probabilistic sensitivity analysis (PSA) and a deterministic sensitivity analysis (DSA) to evaluate the robustness of the results to parameter uncertainty and to investigate their relative contribution to the model's results. The results of the PSA were obtained through 1000 Monte Carlo simulations. Parameter values for these simulations were drawn from the joint distribution of parameters included in the PSA (see Table 1). Vaccination impact was measured using the same set of parameters with and without vaccination for each simulation. We used $95 \%$ confidence intervals (CIs) to present PSA results based on the distribution of each outcome obtained with the simulations performed. The parameters identified and the probability distributions for PSA and one-way DSA are defined in Table 1. The use of triangular distribution for some parameters in the PSA reflects the currently available data or knowledge of the distribution, where only the mode and support values are known or ascertained by experts. We also performed substantial DSA and scenario analysis to highlight the role of specific parameters to complement the PSA.

\section{Results}

\subsection{Model Calibration}

The calibration process yielded a model that was able to reproduce the observed incidence [27] and multiannual cycle of dengue in Malaysia (Fig. 1). The simulated dengue incidence was characterized by an annual variation in dengue incidence without a clear cyclical pattern. There was a period of stable and low incidence that spiked in 2014. The process allowed us to exclude several scenarios that rendered the model incompatible with the observed data, including a short-lived cross-protection Malaysiaspecific expansion factor and average evolution of symptomaticity with age (for primary, secondary and post-secondary infections). The model inherited previous features that reflected the dynamics of dengue transmission, including temporary cross-protection and cross-enhancement. The model also corrected for possible under-reporting from the passive routine surveillance system using an expansion factor that was derived from the control group in 


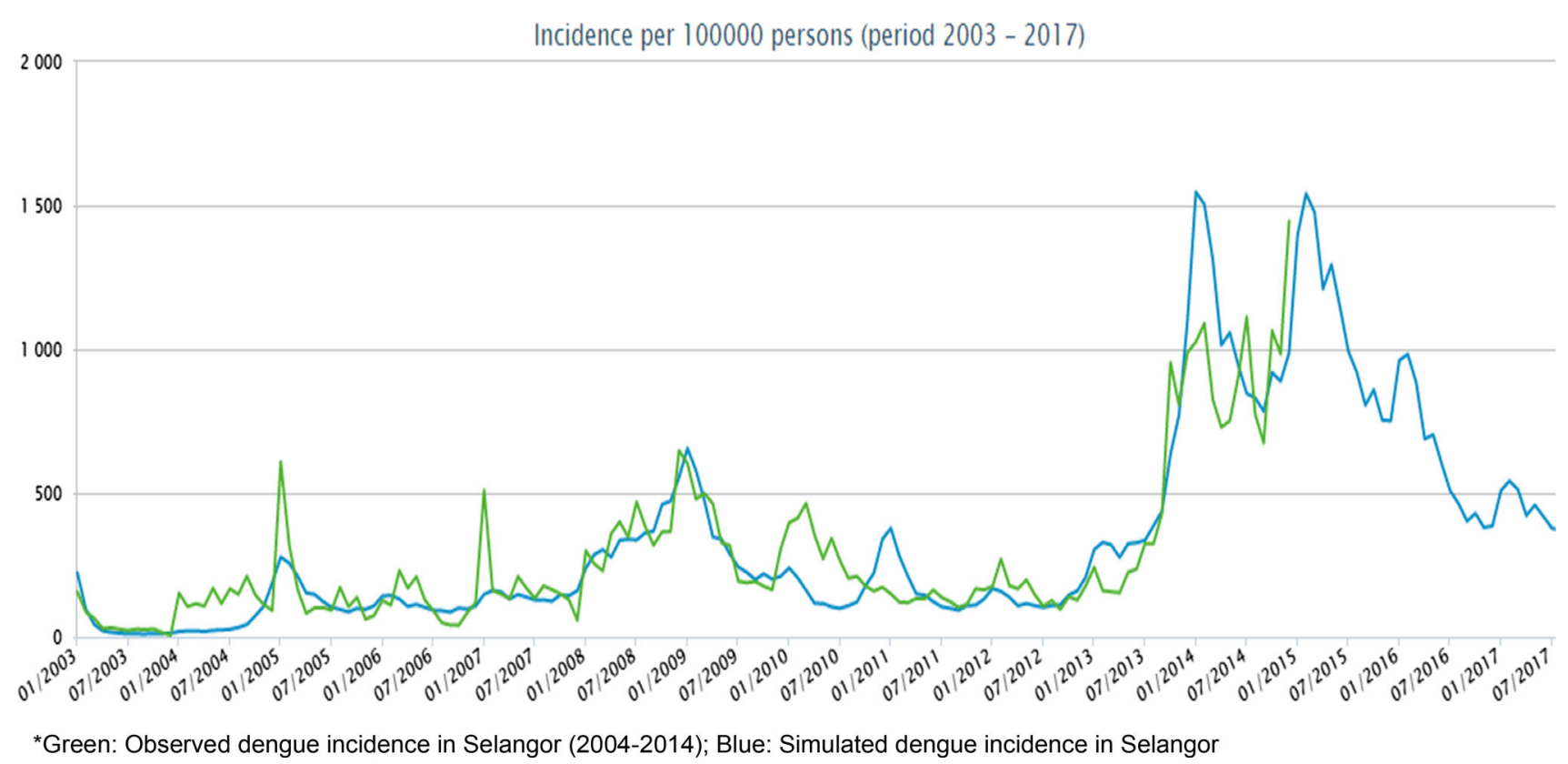

Fig. 1 Observed (green) and projected (blue) numbers of dengue cases in Selangor (2004-2014)

the phase III active surveillance. In the absence of information for adults, the same expansion factors were applied across all ages. These features allow satisfactory characterization of dengue dynamics and a conclusion on the impact of vaccination similar to that in a previous model [28].

\subsection{Vaccination Impact on Disease and Economic Burden}

These results showed that vaccination reduced both disease and economic burden in each of the programmes (Table 2). Programmes 2 (THS R9C30) and 5 (NW R9C30) demonstrated the highest overall impact for each of the geographical groups. However, compared with programmes 1 (THS R13C30) and 4 (NW R13C30), these programmes (where the routine vaccination age was lowered to 9 years with additional catch-up populations) provided only a small additional reduction in overall impact, including total dengue cases $(4.5 \%)$, dengue-related deaths (4.9\%), LYL (4.6\%), DALY (4.8\%) and treatment costs (4.9\%). Programmes 3 and 6 demonstrated the lowest impact across the groups. All health and economic impacts observed with the NW programmes were 2.5 times higher than observed with the THS programmes. Of the DALYs saved with programme 1 , a total of $18.5 \%$ were from hospitalized cases, $23.16 \%$ from ambulatory cases and $58.34 \%$ from dengue-related deaths.

As costs associated with dengue-related deaths were not captured in this study, the total dengue treatment cost was limited to direct and indirect medical costs only. From the provider perspective, it was found that almost two-thirds $(62.50 \%)$ of the cost savings with programme 1 could be attributed to the reduction in ambulatory disease cost and the remaining could be attributed to the reduction in hospitalized disease cost. From the societal perspective, more than half of the cost savings $(56.35 \%)$ with programme 1 was attributed to the savings in productivity costs, $42.09 \%$ of the cost savings were attributed to the reduction in ambulatory disease costs, and the remaining to the reduction in hospitalized disease costs.

\subsection{Cost-Effective Threshold Price and One-Way Deterministic Sensitivity Analysis}

Table 3 shows the cost-effective and highly cost-effective threshold prices from both public provider and societal perspectives. Programme 1 recorded the highest cost-effective threshold prices, at \$US100.59 (95\% CI 72.14-136.74) and \$US127.37 (95\% CI 89.78-175.56) from the provider and societal perspectives, respectively.

The acceptability curves for the cost-effective and highly cost-effective vaccine threshold prices are shown in Fig. 2a-d. The curve demonstrates the cumulative probability of the programmes being cost effective or highly cost effective ( $y$ axis) as a function of vaccine threshold prices defined ( $x$ axis). From the provider perspective, for 90 and $50 \%$ probabilities of the vaccine being highly cost effective, the threshold prices for programme 1 (THS R13C30) were \$US39 and \$US48, respectively, and the threshold prices for programme 4 (NW R13C30) were \$US14 and \$US18, respectively. 


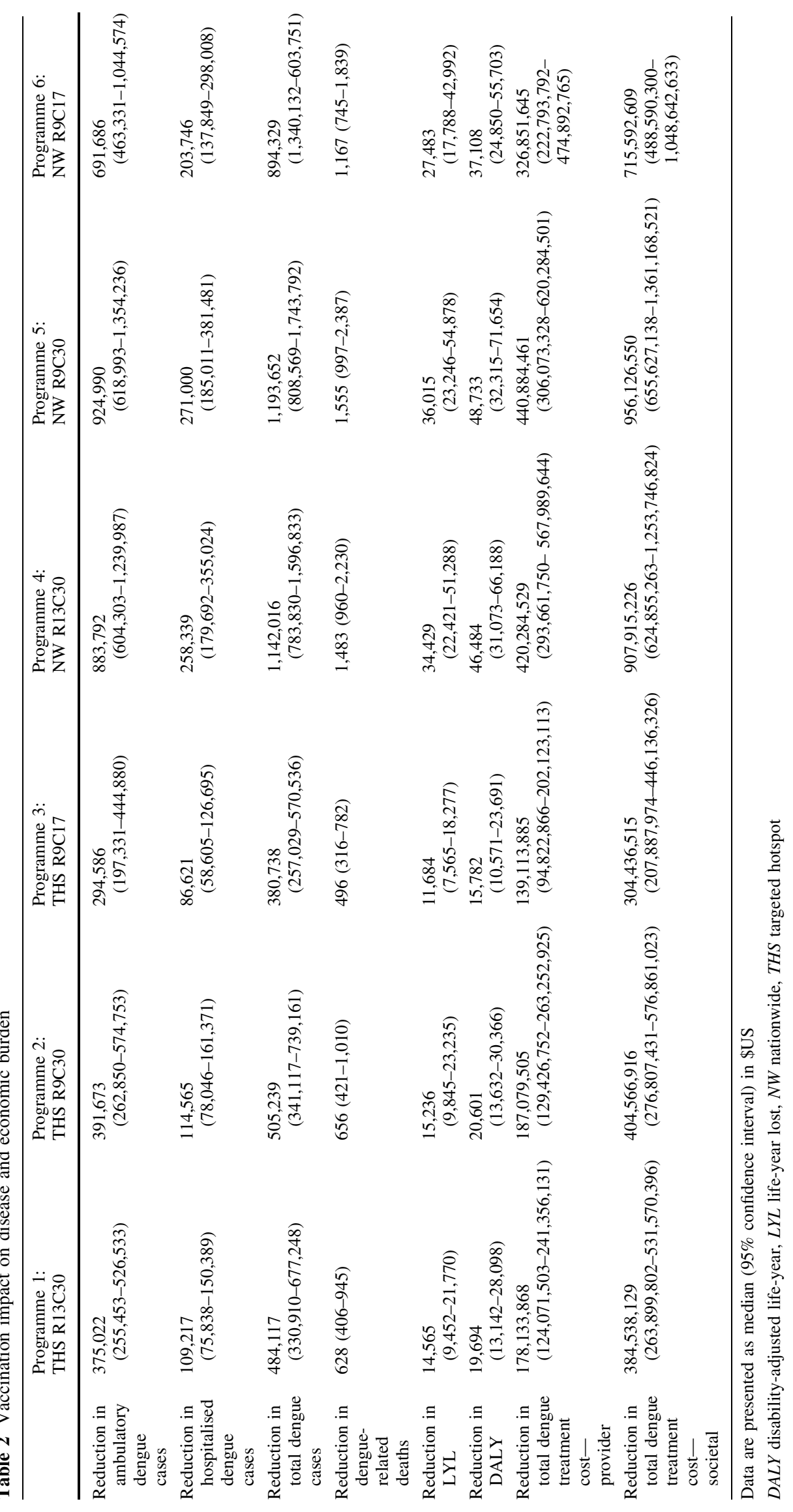


Table 3 Cost-effective threshold price per dose of dengue vaccine

\begin{tabular}{|c|c|c|c|c|}
\hline & \multicolumn{2}{|l|}{ Provider perspective } & \multicolumn{2}{|l|}{ Societal perspective } \\
\hline & Cost effective ${ }^{a}$ & Highly cost effective $^{\mathrm{b}}$ & Cost effective ${ }^{a}$ & Highly cost effective ${ }^{b}$ \\
\hline Programme 1: THS R13C30 & 100.59 (72.14-136.74) & $47.96(33.90-65.21)$ & $123.37(89.78-175.56)$ & $75.02(51.97-103.93)$ \\
\hline Programme 2: THS R9C30 & $91.55(65.34-127.04)$ & $43.52(31.01-59.73)$ & $116.41(81.70-161.46)$ & $68.02(46.82-95.82)$ \\
\hline Programme 3: THS R9C17 & $80.70(56.64-113.62)$ & $37.93(26.39-53.83)$ & $102.26(70.79-145.52)$ & $59.59(40.38-86.85)$ \\
\hline Programme 4: NW R13C30 & $40.76(28.74-56.35)$ & $18.14(12.49-25.68)$ & $50.94(34.76-71.21)$ & $28.60(18.88-40.57)$ \\
\hline Programme 5: NW R9C30 & $36.99(25.92-51.76)$ & $16.43(11.11-23.35)$ & $46.27(31.54-65.41)$ & $25.52(16.64-37.39)$ \\
\hline Programme 6: NW R9C17 & $32.39(22.09-46.33)$ & 14.15 (9.16-21.07) & $40.26(26.90-58.69)$ & $22.01(13.74-33.21)$ \\
\hline
\end{tabular}

Data are presented as median (95\% confidence interval) in \$US

$G D P$ gross domestic product, $N W$ nationwide, THS targeted hotspot

${ }^{\text {a }}$ Cost-effective threshold $=1-3 \times$ Malaysia GDP per capita (\$US10,456.89-31,370.67)

${ }^{\mathrm{b}}$ Highly cost-effective threshold $=<1 \times$ Malaysia GDP per capita $(\$$ US10,456.89)

The vaccine threshold prices were most sensitive to the ambulatory under-reporting factor (Fig. 3a-f). Increasing the ambulatory under-reporting factor to 10.38 increased the highly cost-effective threshold price to \$US82 per dose in programme 1 (from the provider perspective), a $79 \%$ increase compared with the base-case scenario. One-way DSA also showed that vaccine protection duration, hospitalised under-reporting factor and model time horizon exerted a similar impact on the threshold price (Fig. 3a-f). Nevertheless, vaccine administration costs and coverage rates for catch-up cohorts have a negligible impact on the threshold price for all programmes.

\section{Discussion}

Our study assessed the potential health impact, economic impact and cost effectiveness of adding a vaccination programme into the current dengue prevention management in Malaysia with a model that was calibrated with Malaysia-specific epidemiological data. This model successfully reproduced dengue epidemiology in the country and allowed us to simulate the impact of vaccination. The model compared well qualitatively with other models from the WHO Dengue modelling consortium [18]. Model structures and outputs were also found to be generally acceptable by local stakeholders. The stakeholders provided input on the model appropriateness as well as useful insights on the implementation strategies and uncertainties. These processes, actively engaging with multiple stakeholders, increased the model use and credibility at the country level. We designed the vaccine programmes with advice from key MOH personnel and adjusted different coverage and compliance rates for different age groups, geographical locations, routine and catch-up ages and vaccine wastage to accurately reflect the vaccination scenario in Malaysia. This is in line with the recommendation by The Dengue Vaccine Initiative (DVI) expert panels to fill the information gaps related to development and introduction of dengue vaccines [6].

Vaccine benefit is a function of protection conferred to both vaccinated and unvaccinated populations [29]. When a large percentage of the community is vaccinated, the spread of disease is limited. In the context of dengue virus transmission, the indirect effect of vaccine-derived immunity is guided by the reduced transmissibility between host and vector. In this study, vaccination was found to reduce dengue cases over a 10 -year period by almost $50 \%$, similar to the findings of Rodriguez-Barraquer et al. [28]. The biggest reduction occurs within 5 years of vaccination. Differences are due to the effects of the catch-up cohort, which confers rapid but limited protection. Programmes with larger catch-up cohorts exhibited a $56 \%$ reduction in dengue cases, $67-68 \%$ reduction in dengue-related deaths and $68 \%$ reduction in LYL. These results are aligned with the WHO global strategy's goal of reducing dengue mortality by $50 \%$ and morbidity by $25 \%$ by 2020 (with 2010 levels as the baseline) [30]. These reductions would translate into treatment savings ranging from \$US132 to 441 million from the provider perspective over 10 years. This reduction is significant and comparable to human papillomavirus vaccination in Malaysia, where the quadrivalent vaccine was estimated to reduce cervical intraepithelial neoplasia (CIN) cases by $16 \%$ and cervical cancer cases by $81 \%$ [31].

Our results illustrate that programme 1 , which routinely vaccinates those aged 13 years and a catch-up cohort aged 14-30 years in THS areas appears to be the most costeffective programme. With a price of \$US72-136 per dose, dengue vaccine is likely to be cost effective from the provider's perspective. It is likely to be highly cost effective at a price of around \$US34-65 per dose. Its cost- 

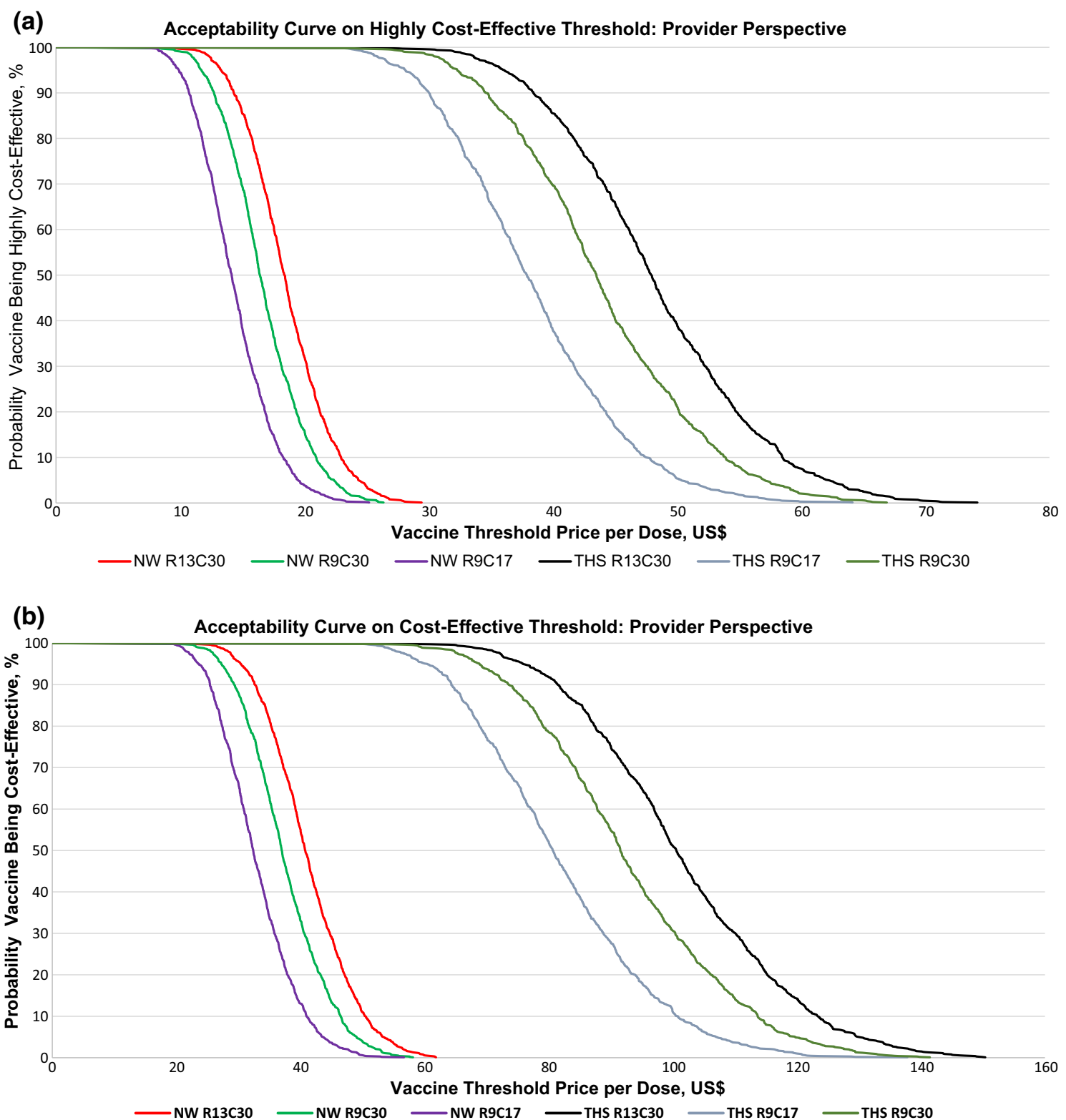

Fig. 2 Cost-effectiveness acceptability curves for a the highly cost-effective vaccine price and $\mathbf{b}$ the cost-effective vaccine price from the provider perspective and for $\mathbf{c}$ the highly cost-effective vaccine price and $\mathbf{d}$ the cost-effective vaccine price from the societal perspective

effective threshold price per vaccine dose from the societal perspective (\$US123.37) was comparable to those in several other countries, e.g. Singapore (\$US95) [9], Thailand (\$US200) [8] and Brazil (\$US534) [10]. While cost data are country specific and cannot be easily compared, it is worth noting that the vaccine administration cost per dose in our study (\$US5.96) was similar to that found in Singapore (\$US7.00) [9]. To account for the opportunity cost, we conducted a threshold analysis on the break-even vaccine price (where net cost equals net benefit). The breakeven price for programme 1 was found to be \$US21.53 (95\% CI 14.81-29.66) per dose from the provider perspective, which is $50 \%$ lower than its highly cost-effective threshold price. This analysis indicated that a price per dose near the break-even threshold would render the vaccination programme cost saving under any circumstances. However, it should be noted that the break-even/costneutral price, where net cost equals net benefit, is not the main objective in this study and is a criterion of lower value as it does not value health gains.

Lastly, our results demonstrated a higher health and economic impact with programmes 2 and 5 but a higher value in terms of cost effectiveness with programmes 1 and 4. This study also found that, from a provider perspective, with additional vaccination for those aged 9-12 years (change from programme 1 to 2), the increased vaccination 
(c) Acceptability Curve on HIghly Cost-Effective Threshold: Societal Perspective

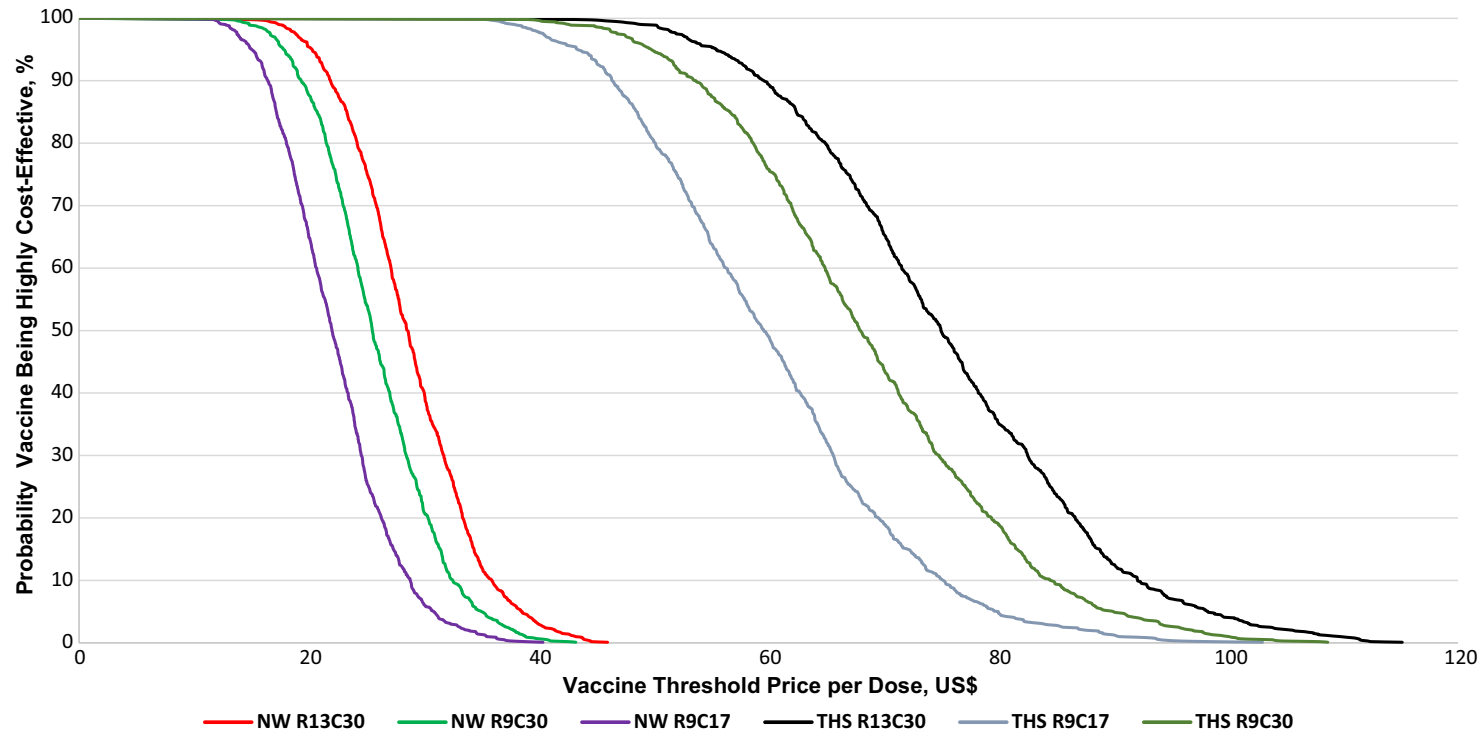

(d)

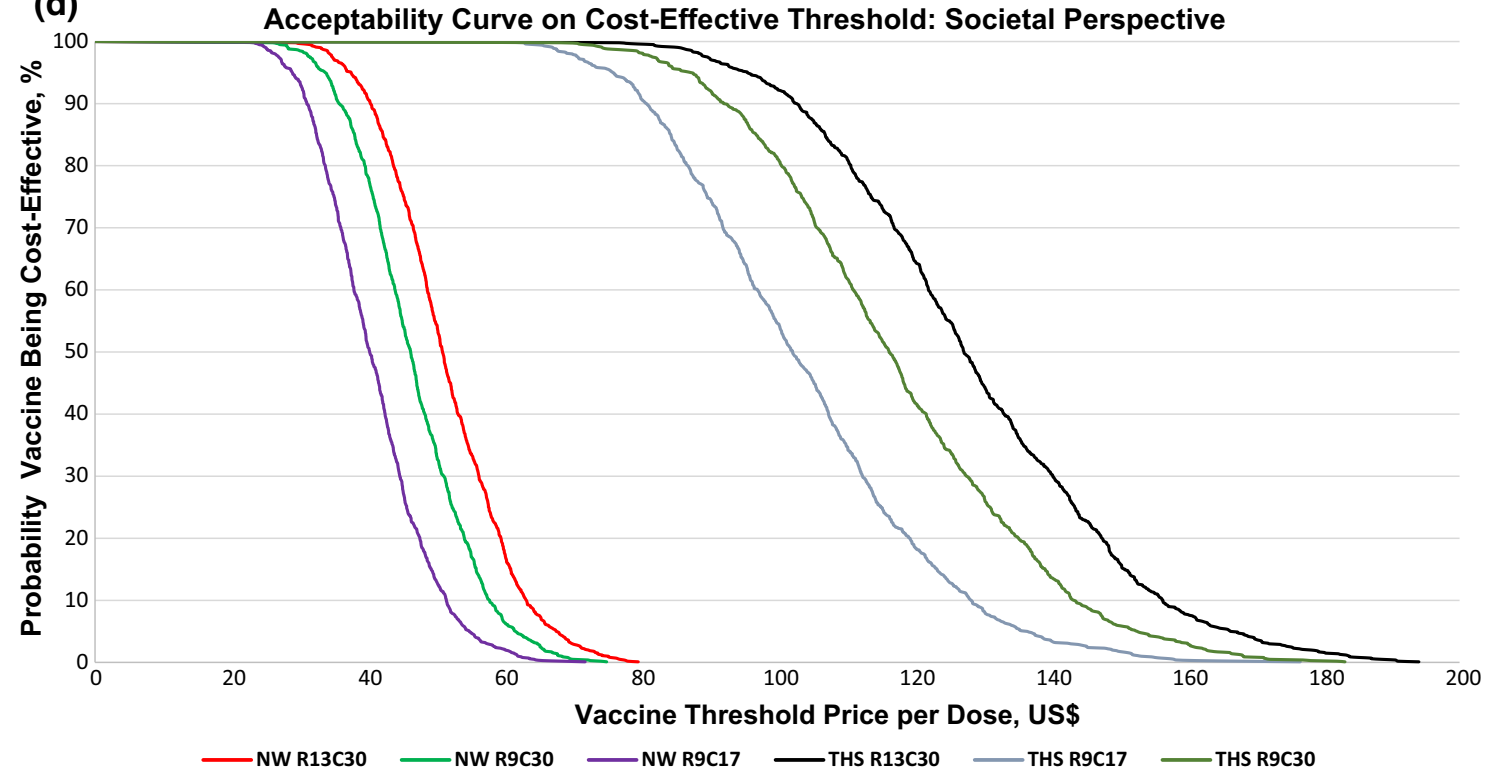

Fig. 2 continued

costs were \$US3.89 million over 10 years, and reducing the catch-up population to age 18 years (change from programme 2 to 3 ) would save \$US4.3 million vaccination costs over the 10-year period. This is consistent with epidemiologic theory, whereby mass prophylactic vaccination could significantly reduce the potential for a major epidemic by reducing the incidence, whereas the targeting of high-risk groups increases efficiency.

Several parameters and model assumptions had a strong influence on the vaccine cost-effective threshold price, particularly under-reporting factors and the duration of vaccine protection, the uncertainty on the latter being a consequence of the relatively short period of longitudinal follow-up available for a vaccine for which the first licensure is dated 2015. The results of one-way DSA in all programmes showed that a higher under-reporting factor and more sustained protection from the vaccine led to more favourable cost-effectiveness results. This is probably caused by the higher health and economic burden curtailed by the vaccine. In programme 1 , the highly cost-effective threshold price of a dose of dengue vaccine can go as low as \$US30-35 assuming no under-reporting in Malaysia. Nevertheless, the estimations of the ambulatory and hospitalised under-reporting 
Fig. 3 One-way sensitivity analysis on the highly costeffective vaccine price from the provider perspective for a programme 1 (THS R13C30), b programme 2 (THS R9C30), c programme 3 (THS R9C17), d programme 4 (NW R13C30), e programme 5 (NW R9C30), f programme $6(\mathrm{NW} \mathrm{R9C17)}$ (a)

Underreporting factors - ambulatory (1/10.38) 30.63

Vaccine protection duration ( $5 / 30$ years)

Model time horizon (5/30 years)

Underreporting factors - hospitalised (1/2.45)

Discount rate $(0 \% / 5 \%)$

Vaccine wastage $(10 \% / 30 \%)$

Total dengue treatment cost ( $80 \% / 120 \%)$ Vaccine coverage rate for catch-up (20\%/80 \%)

Vaccine administration cost (80\%/120\%)

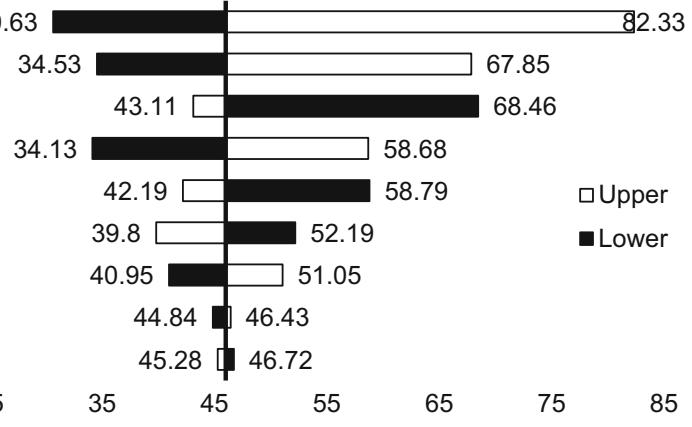

Highly Cost-Effectiveness Threshold Price/dose, US\$

(b)

Underreporting factors - ambulatory (1/10.38)27.14

Vaccine protection duration (5/30 years) 28.31

Underreporting factors - hospitalised (1/2.45) 30.25

Model time horizon (5/30 years)

Discount rate $(0 \% / 5 \%)$

Vaccine wastage $(10 \% / 30 \%)$

Total dengue treatment cost (80\%/120\%)

Vaccine coverage rate for catch up $(20 \% / 80$...

Vaccine administration cost $(80 \% / 120 \%)$

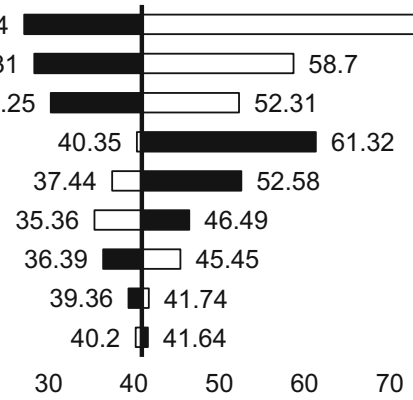

73.72

口Upper

- Lower

Highly Cost-Effectiveness Threshold Price/dose, US\$

(c)

Underreporting factors - ambulatory (1/10.38)25.32

Vaccine protection duration ( $5 / 30$ years)

Underreporting factors - hospitalised (1/2.45)

Model time horizon ( $5 / 30$ years)

Discount rate $(0 \% / 5 \%)$

Vaccine wastage $(10 \% / 30 \%)$

Total dengue treatment cost $(80 \% / 120 \%)$

Vaccine administration cost $(80 \% / 120 \%)$

Vaccine coverage rate for catch up $(90 \ldots$

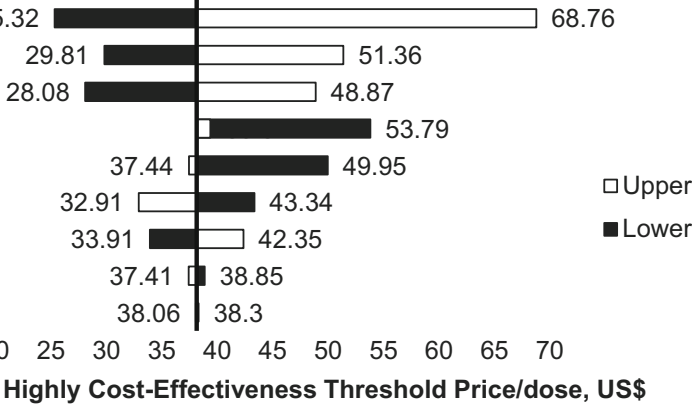

(d)

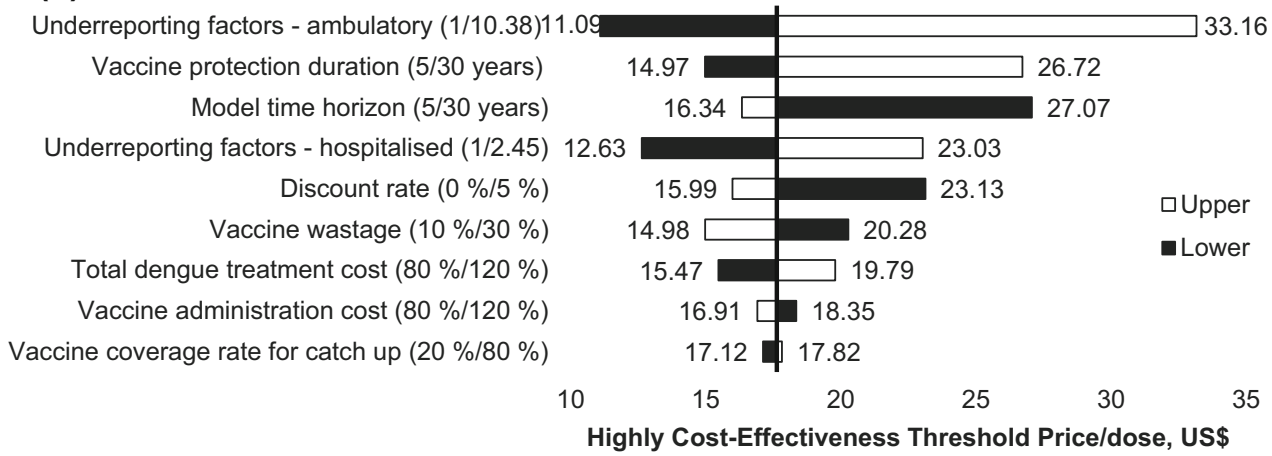

Highly Cost-Effectiveness Threshold Price/dose, US\$ factor in our base case were conservative compared with a neighbouring country with ambulatory and hospitalised under-reporting ranging from 3.8 to 50 and from 1.4 to 3.4 , respectively.
The greatest limitations in our study lie in the presence of uncertainties in the parameters and model assumptions. We did not conduct PSA on all parameters in the model as many of the parameters are correlated and these 
Fig. 3 continued

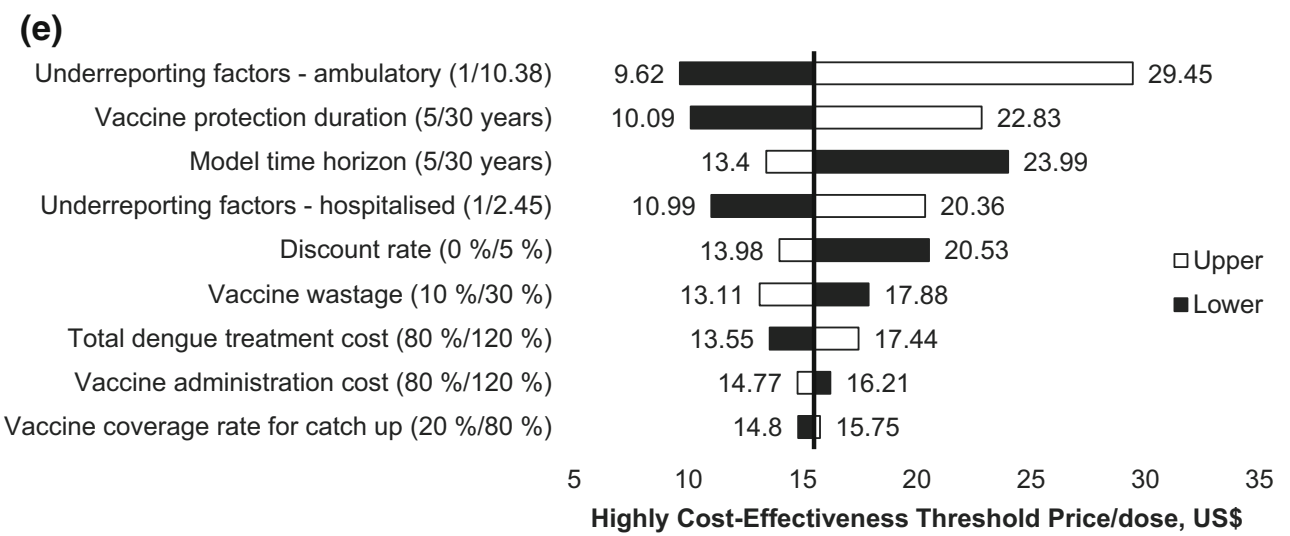

(f)

Underreporting factors - ambulatory (1/10.38)

Vaccine protection duration (5/30 years)

Underreporting factors - hospitalised (1/2.45)

Model time horizon (5/30 years)

Discount rate $(0 \% / 5 \%)$

Vaccine wastage $(10 \% / 30 \%)$

Dengue treatment cost $(80 \% / 120 \%)$

Vaccine administration cost ( $80 \% / 120 \%)$

Vaccine coverage rate for catch up (90\%/100\%)

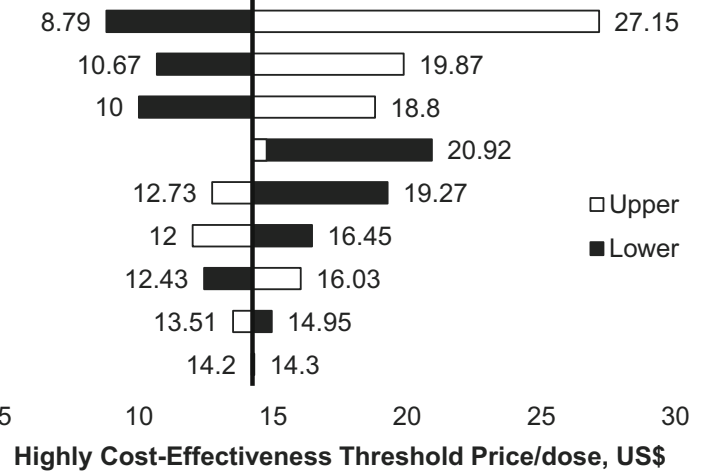

correlations need to be preserved to ensure sensible models and reasonable fit to data, which is in line with the recommendation from the International Society for Pharmacoeconomics and Outcomes Research (ISPOR)-Society for Medical Decision Making (SMDM) Modelling Good Research Practices task force [32]. However, we managed the parameter uncertainties and model assumptions by performing substantial DSA and scenario analysis to highlight the role of specific parameters to complement the PSA. Second, we did not evaluate the effect of waning in immune response and booster vaccine because of the limited follow-up of the long-term efficacy data for which the first licensure was dated December 2015. At the time of writing, the available efficacy data only reported efficacy of pooled data from the first 25 months of two large clinical trials [14-16]. Third, the dengue treatment cost data in this study were conservative as they were limited to direct and indirect medical costs only. Other costs associated with dengue-related deaths, long-term sequelae of dengue disease, vector prevention and control, surveillance, loss in tourism and loss in foreign investment were not included but are expected to increase the benefit (and value) of the vaccine. However, this is in line with other available dengue modelling studies [7-10]. Finally, because of the absence of country-specific cost-effectiveness thresholds in Malaysia, we reported the cost effectiveness with reference to the thresholds set by WHO-CHOICE (Choosing Interventions that are cost-effective). Although the WHOCHOICE thresholds are widely cited and used to assess public health measures or immunization programmes in low and middle-income countries (LMICs) [33, 34] and recommended by WHO in countries without locally established thresholds [35], there is debate on its appropriateness as it does not reflect the budget affordability of the programme being evaluated. In our study, we addressed this issue by comparing the break-even price (i.e. no net cost) with the WHO-CHOICE threshold price. In fact, recent published studies exploring the willingness to pay (WTP) per quality-adjusted life-year (QALY) in Malaysia showed that the threshold ( $\sim$ US9000) is not very far from the $1 \times$ GDP threshold (\$US10,456) [36, 37].

To date, only a few published models [7-9] have evaluated the economic value of dengue vaccines. Three of them were static models that did not consider the benefits of herd immunity, which might have underestimated the cost effectiveness of the vaccine. One model [10] evaluated the cost effectiveness of the vaccine using an age-structured four-serotype dengue transmission and vaccination model. However, none of the available models incorporated the Malaysia-specific demographic and epidemiologic data. In addition, new clinical evidence in long-term vaccine efficacy and safety have emerged that warrant an 
improved evaluation of the economic value of a dengue vaccine in Malaysia.

All vaccination programmes evaluated in our study demonstrated positive health and economic impacts. It is important to consider vaccination as a long-term investment, as its full benefit will only be observed years after its introduction when the higher initial costs due to catch-up vaccination are offset by the morbidity and mortality averted.

\section{Conclusions}

Our study shows that routine vaccination in a population aged 13 years with a catch-up cohort aged 14-30 years in THS areas is the best-value strategy among the six strategies investigated. Vaccination against dengue disease could complement the existing surveillance and vector control in curbing the economic and health burden of the disease. The robustness of the results in our study suggests that dengue vaccination is potentially a good investment if the purchaser could negotiate a price below the determined costeffective threshold price for the programmes evaluated.

Data availability statements Several datasets were used for this analysis: (1) Data collected during phase III efficacy trials in ten countries were used to estimate the parameters of the mathematical model related to dengue transmission, interactions between serotypes and vaccine efficacy. The entire dataset is not publicly available, but the results of these trials have been presented in several publications. (2) Data from dengue surveillance in Malaysia were used to adapt the model to the Malaysian situation. These data are available from the corresponding author upon request. (3) Data related to dengue costs in Malaysia were obtained from other publications that present the original datasets used for deriving these costs. (4) The model has been developed to be run through a web-based interface (DENMOD) accessible to registered users. The access to this interface can be obtained upon request.

Acknowledgements The authors thank the Director General of Health Malaysia for his permission to publish this article. We also acknowledge the technical input and constructive suggestions from the following experts: Dr. Chee Kheong Chong and Dr. Rose Nani Mudin from Disease Control Division, MOH Malaysia. We acknowledge Nicola Truss, inScience Communications, Springer Healthcare, who provided proofreading assistance. This assistance was funded by Sanofi Pasteur.

Author contributions AAS and HYY designed the study. LC constructed the model. BSG, RJ and A-SHSS advised on the parameterization of the model construction. LS made substantial contribution in the acquisition of data. AAS and HYY assembled the data and did the statistical analysis. HYY wrote the first draft and all authors contributed to further drafts and approved the final manuscript.

\section{Compliance with ethical standards}

Funding This study was supported by an agreement between SanofiAventis Singapore and Universiti Sains Malaysia.

Conflict of interest HYY and AAS have received research grants from Sanofi-Aventis Singapore Ptd. Lte. LC and LS are employees of Sanofi-Pasteur. BSG and RJ have no conflicts of interest. A-SHSS has received consultation honorarium from Sanofi-Aventis Singapore Ptd. Lte.

Ethical approval This article does not contain any studies with human participants or animals performed by any of the authors.

\section{References}

1. Bhatt S, Gething PW, Brady OJ, Messina JP, Farlow AW, Moyes $\mathrm{CL}$, et al. The global distribution and burden of dengue. Nature. 2013;496(7446):504-7. doi:10.1038/nature12060.

2. World Health Organization. Dengue Situation Updates. http:// www.wpro.who.int/emerging_diseases/DengueSituationUpdates/ en/. Accessed 11 Oct 2016

3. Shepard DS, Undurraga EA, Lees RS, Halasa Y, Lum LC, Ng $\mathrm{CW}$. Use of multiple data sources to estimate the economic cost of dengue illness in Malaysia. Am $J$ Trop Med Hyg. 2012;87(5):796-805. doi:10.4269/ajtmh.2012.12-0019.

4. Undurraga EA, Halasa YA, Shepard DS. Use of expansion factors to estimate the burden of dengue in southeast Asia: a systematic analysis. PLoS Negl Trop Dis. 2013;7(2):e2056. doi:10.1371/ journal.pntd.0002056.

5. Horstick O, Runge-Ranzinger S, Nathan MB, Kroeger A. Dengue vector-control services: how do they work? A systematic literature review and country case studies. Trans R Soc Trop Med Hyg. 2010;104(6):379-86. doi:10.1016/j.trstmh.2009.07.027.

6. Beatty ME, Beutels P, Meltzer MI, Shepard DS, Hombach J, Hutubessy R, et al. Health economics of dengue: a systematic literature review and expert panel's assessment. Am J Trop Med Hyg. 2011;84(3):473-88. doi:10.4269/ajtmh.2011.10-0521.

7. Shepard DS, Suaya JA, Halstead SB, Nathan MB, Gubler DJ, Mahoney RT, et al. Cost-effectiveness of a pediatric dengue vaccine. Vaccine. 2004;22(9):1275-80.

8. Lee BY, Connor DL, Kitchen SB, Bacon KM, Shah M, Brown ST, et al. Economic value of dengue vaccine in Thailand. Am J Trop Med Hyg. 2011;84(5):764-72. doi:10.4269/ajtmh.2011.100624.

9. Carrasco LR, Lee LK, Lee VJ, Ooi EE, Shepard DS, Thein TL, et al. Economic impact of dengue illness and the cost-effectiveness of future vaccination programs in Singapore. PLoS Negl Trop Dis. 2011;5(12):e1426. doi:10.1371/journal.pntd.0001426.

10. Durham DP, Ndeffo Mbah ML, Medlock J, Luz PM, Meyers LA, Paltiel AD, et al. Dengue dynamics and vaccine cost-effectiveness in Brazil. Vaccine. 2013;31(37):3957-61. doi:10.1016/j. vaccine.2013.06.036.

11. Shim E. Dengue dynamics and vaccine cost-effectiveness analysis in the Philippines. Am J Trop Med Hyg. 2016;16:0194.

12. Coudeville L, Garnett GP. Transmission dynamics of the four dengue serotypes in southern Vietnam and the potential impact of vaccination. PLoS One. 2012;7(12):e51244. doi:10.1371/journal. pone.0051244. 
13. Amar-Singh HSS, Koh M-T, Tan KK, Chan LG, Zhou L, Bouckenooghe A, et al. Safety and immunogenicity of a tetravalent dengue vaccine in healthy children aged 2-11 years in Malaysia: a randomized, placebo-controlled, phase III study. Vaccine. 2013;31(49):5814-21. doi:10.1016/j.vaccine.2013.10. 013.

14. Villar L, Dayan GH, Arredondo-Garcia JL, Rivera DM, Cunha R, Deseda $C$, et al. Efficacy of a tetravalent dengue vaccine in children in Latin America. N Engl J Med. 2015;372(2):113-23. doi:10.1056/NEJMoa1411037.

15. Capeding MR, Tran NH, Hadinegoro SRS, Ismail HIHJM, Chotpitayasunondh T, Chua MN, et al. Clinical efficacy and safety of a novel tetravalent dengue vaccine in healthy children in Asia: a phase 3, randomised, observer-masked, placebo-controlled trial. Lancet. 2014;384(9951):1358-65. doi:10.1016/ S0140-6736(14)61060-6.

16. Hadinegoro SR, Arredondo-García JL, Capeding MR, Deseda C, Chotpitayasunondh T, Dietze R, et al. Efficacy and long-term safety of a dengue vaccine in regions of endemic disease. $\mathrm{N}$ Engl J Med. 2015;373(13):1195-206. doi:10.1056/NEJMoa1506223.

17. World Health Organization. Summary of the April 2016 meeting of the Strategic Advisory Group of Experts on immunization (SAGE). Geneva: WHO; 2016. p. 02016.

18. World Health Organization. Comparative modelling of dengue vaccine public health impact (CMDVI). Geneva: WHO; 2016.

19. Mohd-Zaki AH, Brett J, Ismail E, L'Azou M. Epidemiology of dengue disease in Malaysia (2000-2012): a systematic literature review. PLoS Negl Trop Dis. 2014;8(11):e3159. doi:10.1371/ journal.pntd.0003159.

20. UNICEF. State of the world's children 2015 country statistical information. New York: UNICEF; 2013. http://www.unicef.org/ infobycountry/malaysia_statistics.html. Accessed 30 July 2015.

21. World Health Organization. Managing vaccine wastage at country level: guidelines for programme managers. Geneva: WHO; 2003.

22. Suaya JA, Shepard DS, Siqueira JB, Martelli CT, Lum LC, Tan $\mathrm{LH}$, et al. Cost of dengue cases in eight countries in the Americas and Asia: a prospective study. Am J Trop Med Hyg. 2009;80(5):846-55.

23. Shepard DS, Undurraga EA, Halasa YA. Economic and disease burden of dengue in southeast Asia. PLoS Negl Trop Dis. 2013;7(2):e2055. doi:10.1371/journal.pntd.0002055.

24. International Monetary Fund. World Economic Outlook Database. http://www.imf.org/external/pubs/ft/weo/2014/02/weodata/ index.aspx. Accessed 15 Dec 2014.

25. Malaysia Ministry of Health. Pharmacoeconomic guideline for Malaysia. Putrajaya: MoH; 2012.

26. Commission on Macroeconomics and Health. Macroeconomics and health: investing in health for economic development. Report of the Commission on Macroeconomics and Health. Geneva: World Health Organization; 2001.

27. Malaysia Ministry of Health. Age-specific annual dengue incidence in Selangor 2003-2013. Putrajaya: MoH; 2014.

28. Rodriguez-Barraquer I, Mier-y-Teran-Romero L, Schwartz IB, Burke DS, Cummings DA. Potential opportunities and perils of imperfect dengue vaccines. Vaccine. 2014;32(4):514-20.
29. Halloran ME, Haber M, Longini IM, Struchiner CJ. Direct and indirect effects in vaccine efficacy and effectiveness. Am J Epidemiol. 1991;133(4):323-31.

30. World Health Organization. Global strategy for dengue prevention and control, 2012-2020 Report no. WHO/HTM/NTD/VEM/ 2012.5. Geneva: WHO; 2012.

31. Aljunid S, Zafar A, Saperi S, Amrizal M. Burden of disease associated with cervical cancer in Malaysia and potential costs and consequences of HPV vaccination. Asian Pac J Cancer Prev. 2010;11(6):1551-9.

32. Pitman R, Fisman D, Zaric GS, Postma M, Kretzschmar M, Edmunds J, et al. Dynamic transmission modeling: a report of the ISPOR-SMDM modeling good research practices task force-5. Value Health. 2012;15(6):828-34.

33. Tu HA, Woerdenbag HJ, Kane S, Rozenbaum MH, Li SC, Postma MJ. Economic evaluations of rotavirus immunization for developing countries: a review of the literature. Expert Rev Vaccines. 2011;10(7):1037-51. doi:10.1586/erv.11.65.

34. Fesenfeld M, Hutubessy R, Jit M. Cost-effectiveness of human papillomavirus vaccination in low and middle income countries: a systematic review. Vaccine. 2013;31(37):3786-804. doi:10. 1016/j.vaccine.2013.06.060.

35. Walker DG, Hutubessy R, Beutels P. WHO guide for standardisation of economic evaluations of immunization programmes. Vaccine. 2010;28(11):2356-9.

36. Shafie AA, Lim YW, Chua GN, Hassali MAA. Exploring the willingness to pay for a quality-adjusted life-year in the state of Penang, Malaysia. Clinicoecon Outcomes Res. 2014;6:473-81. doi:10.2147/CEOR.S67375.

37. Lim YW, Shafie AA, Chua GN, Hassali M. Determination of cost-effectiveness threshold for Malaysia. George Town: Universiti Sains Malaysia; 2015.

38. Department of Statistics Malaysia. Population distribution and basic demographic characteristic report 2010. Putrajaya: DoS; 2011.

39. Salomon JA, Wang H, Freeman MK, Vos T, Flaxman AD, Lopez AD, et al. Healthy life expectancy for 187 countries, 1990-2010: a systematic analysis for the Global Burden Disease Study 2010. Lancet. 2013;380(9859):2144-62.

40. World Health Organization. Choosing interventions that are cost effective (WHO-CHOICE). Geneva: WHO; 2011. http://www. who.int/choice/cost-effectiveness/inputs/health_service/en/.

41. Guidelines on the Implementation of the Minimum Wages Order 2012. In: Putrajaya, editor. Ministry of Human Resources. National Wages Consultative Council; 2012.

42. Ministry of Health Malaysia. Handbook of National Immunization Programme for Babies and Children (for Nurses). Putrajaya: Family Health Promotion Division; 2008.

43. Coudeville L, Baurin N, Vergu E. Estimation of parameters related to vaccine efficacy and dengue transmission from two large phase III studies. Vaccine. 2015. doi:10.1016/j.vaccine. 2015.11.023.

44. Nealon JTA, Capeding MR, Tran NH, Hadinegoro SR, Chotpitayasunondh T, et al. Symptomatic dengue burden in 5 countries in Asia-Pacific: epidemiological evidence from a dengue vaccine trial. In: 5th Asian vaccine conference, Hanoi; 2015. 